\section{ORIGINAL RESEARCH}

\section{G. Spilberg}

S.L. Carniato

R.M. King

I.M.J. van der Bom

M. Mehra

R.P. Walvick

A.K. Wakhloo

M.J. Gounis

\title{
Temporal Evolution of Susceptibility Artifacts from Coiled Aneurysms on MR Angiography: An In Vivo Canine Study
}

\begin{abstract}
BACKGROUND AND PURPOSE: Intracranial aneurysms treated by coiling have a risk for recurrence, requiring surveillance imaging. MRA has emerged as an attractive technique for postcoiling aneurysm imaging. Previous research has evaluated MR imaging artifacts of the coil mass in vitro. Our aim in this study was to evaluate MR imaging artifacts of coiled aneurysms in vivo with time.
\end{abstract}

\begin{abstract}
MATERIALS AND METHODS: Four sidewall aneurysms were created in each of 4 dogs. Aneurysms were embolized receiving only 1 type of coils. After embolization, the animals were transferred to MR imaging, which included axial 3D TOF MRA (TEs, 3.5, 5, and $6.9 \mathrm{~ms}$ ), phase-contrast MRA, and coronal CE-MRA. MR imaging studies were repeated at 1, 4, 6, 8, 14, and 28 weeks. We calculated an OEF: $\mathrm{OEF}=\mathrm{V}_{\mathrm{A}} \mathrm{N}_{\mathrm{CM}}$, where the numerator represents the volume of the MR imaging artifacts and the denominator is the true volume of the coil mass measured by 3D RA.
\end{abstract}

RESULTS: OEFs were largest immediately after embolization and showed a gradual decay until approximately 4 weeks, when there was stabilization of the size of the artifacts. By 4 weeks, there was mild coil compaction (average coil mass volume decrease of $7.8 \%$ ); however, the OEFs decreased by $25 \%$ after 4 weeks $(P<.001)$.

CONCLUSIONS: MR imaging susceptibility artifacts change with time, being maximal in the postembolization setting and decaying until 4 weeks. The clinical implications of this study are that baseline MRA for comparison with future imaging should be acquired at a minimum of 1 week after the procedure.

ABBREVIATIONS: $\mathrm{ACT}=$ activated clotting time; $\mathrm{CCA}=$ common carotid artery; $\mathrm{CE}=$ contrastenhanced; $\mathrm{CuSO}_{4}=$ copper sulphate; $3 \mathrm{D} \mathrm{RA}=3 \mathrm{D}$ rotational angiography; $\mathrm{GDC}=$ Guglielmi detachable coil; $\mathrm{OEF}=$ overestimation factor; $\mathrm{SEM}=$ scanning electron microscopy; $\mathrm{TE}=$ echo time; TOF = time-of-flight

E ndovascular procedures are increasingly being used for the treatment of intracranial aneurysms. In this setting, coiling has evolved with new devices and technologies achieving better outcomes. ${ }^{1}$ Nevertheless, recurrence due to coil compaction and/or aneurysm regrowth still remains a limitation of the method, and long-term imaging follow-up is a requirement. ${ }^{2-5}$

DSA is an invasive procedure that involves risks for permanent neurologic deficits ranging from $0 \%$ to $5.7 \%{ }^{6-8}$ and also exposure to ionizing radiation, which has been recently regarded with increasing concern, especially in this subset of patients who receive high doses from diagnostic work-up and treatment procedures. ${ }^{9}$ CTA is very effective in detecting an-

Received May 25, 2011; accepted after revision June 27.

From the Department of Radiology (G.S., R.M.K., I.M.J.v.d.B., M.M., A.K.W., M.J.G.), New England Center for Stroke Research, University of Massachusetts Medical School, Worcester, Massachusetts; Research and Development (S.L.C.), Stryker Neurovascular, Fremont California; and Department of Radiology (R.P.W.), New York University School of Medicine, New York, New York.

This work was supported by Stryker Neurovascular. We also acknowledge technology support from the National Center for Research Resources Award Number S10RR027897 and Phillips Healthcare. The content is solely the responsibility of the authors and does not necessarily represent the official views of the sponsoring agencies.

Please address correspondence to Matthew J. Gounis, PhD, Department of Radiology, University of Massachusetts, New England Center for Stroke Research, 55 Lake Ave N, SA-107R, Worcester, MA 01655; e-mail: matt.gounis@umassmed.edu

Indicates open access to non-subscribers at www.ajnr.org

Indicates article with supplemental on-line table.

http://dx.doi.org/10.3174/ajnr.A2831 eurysms; however, the beam-hardening and streak artifacts limit its use as a posttreatment imaging method. ${ }^{10}$ Recently, MRA has become a viable option for noninvasive follow-up. 3D-TOF and CE-MRA offer a noninvasive high-resolution alternative to DSA for postembolization surveillance due to its high flow sensitivity and lack of ionizing radiation. Other advantages include comfort, convenience for the patient, and cost reduction. ${ }^{10-13}$ Often, a baseline MRA within 1 week of coiling is acquired for future comparison. This timeframe is presumably based on the hospital admission of the patient.

With MR imaging, it is possible to obtain information on aneurysm residual patency or recanalization and parent vessel abnormalities. The coil mass produces magnetic susceptibility or blooming artifacts that can obscure visualization of these parameters and subsequent interpretation. Shorter TE techniques can be used to reduce susceptibility-induced signalintensity loss from the coil mass. ${ }^{14,15}$ Previous research into MR imaging artifacts has been conducted mostly in vitro, ${ }^{16-19}$ but the dynamic characteristics of a functional vascular system cannot be fully simulated. Plastic or vascular replicas have little MR imaging signal intensity compared with the arterial wall. Most important, the signal intensity from the aneurysm healing process - the acute thrombosis and thrombus organization-are not evaluated. Therefore, implantation and imaging of these devices in animal models are desirable. Our aim in this study was to evaluate the MR imaging artifacts of the coiled aneurysms in an animal model in different MRA sequences, with time. 


\section{Materials and Methods}

For this study, we selected the venous pouch aneurysm in the canine model. ${ }^{20}$ The model was selected because multiple aneurysms can be created within the same animal, thus permitting a reduction of the number of animals needed. Furthermore, because the aneurysms are located in the neck region, respiratory gating for MR imaging is not necessary. This model has been well-described in the literature as a suitable one for the evaluation of emerging endovascular devices for aneurysmal therapy. ${ }^{21,22}$

\section{In Vivo Experiments}

Anesthesia, Analgesia, and Antibiotic Regimens. All animal experiments were performed in accordance with a protocol approved by our Institutional Animal Care and Use Committee. All procedures were performed with the animal under general anesthesia and by using strict aseptic techniques. Before all surgical or imaging procedures, the animals were preanesthetized by an intramuscular injection of acepromazine $(0.06 \mathrm{mg} / \mathrm{kg})$ and glycopyrrolate $(0.01 \mathrm{mg} / \mathrm{kg})$. Anesthesia was induced by an IV injection of thiopental $(15 \mathrm{mg} / \mathrm{kg})$ or propofol $(3 \mathrm{mg} / \mathrm{kg})$ and was maintained with mechanical ventilation of $1 \%-4 \%$ isoflurane. The physiologic status of the animal was assessed by using continuous monitoring of respiration rate, heart rate, oxygen saturation level, end-tidal $\mathrm{CO}_{2}$ level, and temperature. Before surgical procedures, the animals were given buprenorphine $(0.02 \mathrm{mg} /$ $\mathrm{kg}$, subcutaneous) and a fentanyl patch was applied (50 $\mu \mathrm{g} / \mathrm{h}$, transdermal, 3 days). During the surgical intervention, cefazolin $(20 \mathrm{mg} /$ $\mathrm{kg}$, IV) was administered and repeated every 2 hours until closure for prophylactic infection control.

Aneurysm Creation. Four mongrel canines (female; mean weight, $16.5 \mathrm{~kg}$ ) were used to investigate the aims of the study. The details of the venous pouch surgical aneurysm construction have been available for decades and are described elsewhere. ${ }^{20}$ Briefly, 2 venous sidewall aneurysms were created on each CCA for a total of 4 aneurysms in each animal. Two arteriotomies were made in each CCA, followed by an end-to-side anastomosis of a resected portion of the external jugular vein to the arteriotomy site. The top of the vein pouch was ligated at a linear distance of between 4 and $6 \mathrm{~mm}$ from the parent artery. Before closing the incision, we performed a transfemoral conventional angiography of both CCAs to confirm aneurysm and artery patency.

Interventional Procedure. After allowing the aneurysms to heal for a minimum of 2 weeks, the animals were prepared for the coiling procedure. The left inguinal region of the animal was prepared for a femoral cut-down followed by placement of a $6 \mathrm{~F}$ hemostatic introducer in the left femoral artery by using a modified Seldinger technique. The introducer was secured in place, with the distal aspect of the femoral artery ligated. Baseline ACT was measured, and a loading dose of IV heparin $(75 \mathrm{IU} / \mathrm{kg}$ ) was administered to maintain ACT levels above approximately twice that recorded at baseline. A $6 \mathrm{~F}$ guide catheter was placed through the introducer and navigated past the origin of the CCA in preparation for precoiling $3 \mathrm{D}$ and planar angiography. Measurements were taken to characterize the implant sites on the basis of the dimensions of the aneurysm and the parent vessel diameter.

Aneurysms were randomly selected to be embolized with GDCs (Stryker Neurovascular, Fremont, California) or Target detachable coils (Stryker Neurovascular). A 2-tipped Excelsior SL-10 microcatheter (Stryker Neurovascular) was advanced into the neck of the aneurysm in preparation for coil embolization. Coils were deployed and detached in accordance with the representative instructions for use of each coil. Aneurysms were embolized by receiving only 1 family of coils. For each aneurysm coiled with the Target coils, another aneurysm was coiled with the GDC coils by using the identical number of coils, size, and type.

MR Imaging. Following completion of the aneurysm coiling, the animals were transferred to 3T MR imaging (Achieva3T; Philips Healthcare, Best, the Netherlands). MR imaging was performed on animals in dorsal recumbency, head first into the magnet. The neck of the animal was placed into an 8-element receive-only knee coil. The MR imaging technique included coronal T1 unenhanced turbo spin-echo, axial 3D-TOF MRA (TEs of 3.5, 5, and 6.9), and phasecontrast MRA (On-line Table). Animals were returned to the MR imaging center at $1,4,6,8,14$, and 28 weeks after coil embolization for repeat imaging studies as previously performed, with the addition of coronal CE-MRA sequences. The in-plane resolution on the MR angiography sequences was approximately $0.4 \mathrm{~mm}$, and section thickness, $0.7 \mathrm{~mm}$. Gadobenate dimeglumine $(0.1 \mathrm{mmol} / \mathrm{kg}$, IV $)$ was administered before CE-MRA with bolus tracking; namely, the sequence was commenced on visualization of contrast entering the ascending aorta. Total MR imaging protocol was approximately 30 minutes.

Quantitative Analysis of Susceptibility Artifacts. At each imaging time point, a set of 3D RA images (FOV, $250 \times 250 \times 250 \mathrm{~mm}$; isotropic voxel size, $0.98 \mathrm{~mm}$ ) was acquired by using an $\mathrm{x}$-ray angiography C-arm system (Allura Xper FD20, Philips Healthcare) to measure the coil mass volume. This method has been shown to reliably determine aneurysm volume $\mathrm{e}^{23}$ and is readily applied to measure the volume of the highly radiopaque coil mass. 3D RA measurements were all performed by the same blinded operator, in the same fashion, on dedicated software (XtraVision Release 8.1.1.10012, Philips Healthcare). The measurements were carried out by using a semiautomated method based on local image intensities in the following sequence: 1) The coil mass was isolated by the volumemeasuring tool. 2) The threshold was set to the automatic value. 3) The window was set to $100 \%$; thereby creating a binary volume. This was done because the volume-measuring tool uses this setting to determine the volume; changing the contrast has no effect on the volume. 4) The level was visually adjusted to reduce streak artifacts. Because of the relative high intensities caused by the coils, this threshold value was very reproducible with $<5 \%$ variation between experiments.

To measure artifact volume, MRA sequences were exported to a DICOM file and analyzed in Mimics 13.1 (Materialise, Leuven, Belgium). Once the MR images were imported into Mimics, we chose 2 axial sections: 1 from the proximal set of aneurysms and the other from the distal aneurysms. In each of the sections, a small region of interest measuring $5 \mathrm{~mm}^{2}$ was selected adjacent to (not including) the susceptibility artifacts. Within this small region of interest, the average gray value was calculated. As per the American Society for Testing and Materials 2119-07 guidelines (http://www.astm.org/Standards/ F2119.htm) for MR imaging testing of new devices, a value of $>30 \%$ signal-intensity change was set as artifact. This value was selected as a threshold for outlining the area of susceptibility artifact, and any voxels with a gray value less than this were considered artifact. Manual regions of interest were drawn that encompassed the area of artifact. This manual segmentation was used to limit subsequent thresholding, thereby ensuring that small vessels close to the artifact were not incorporated into the artifact volume measurements. The artifact areas were then reconstructed into 3D volumes, and their volume was mea- 


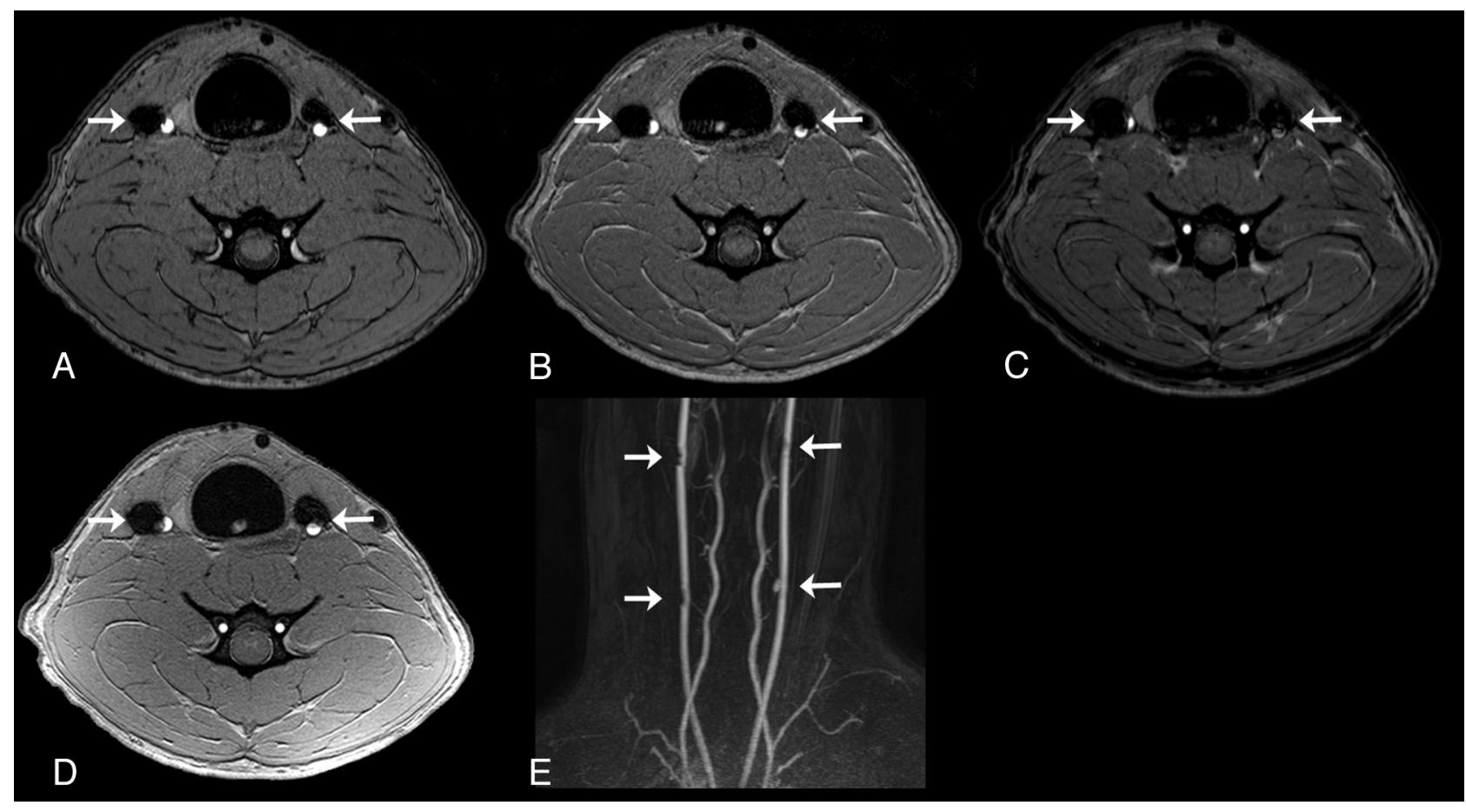

Fig 1. Representative MRA images at 8 weeks postembolization. Axial TOF-MRA images with TEs of $3.5(A), 5(B)$, and 6.9 ms $(C)$ of distal aneurysms $($ arrows $)$ in the same subject. Note the increase of artifact size as the TE increases. D, Axial images from a phase-contrast MRA sequence of the same aneurysms (arrows). E, CE-MRA (coronal, maximum-intensity-projection reconstruction) of all 4 aneurysms (arrows) in a single subject.

sured in cubic millimeters. The OEF was calculated from this volume ${ }^{17}$ :

$$
\mathrm{OEF}=\mathrm{v}(\operatorname{artifact}) / \mathrm{v}(\text { coil mass })
$$

\section{In Vitro Experiments}

To further understand the MR imaging artifacts, we performed a series of in vitro experiments. We created a $\mathrm{CuSO}_{4}$ gelatin phantom with 4 spheric cavities filled with $0.9 \%$ saline; 2 with 3 - and 2 with 4 -mm radii. To create the phantom, we had a 2-step process: 3 -and 4-mm radii spheres were inserted in semiliquid gelatin cups. After the gelatin solidified, spheres were gently extracted from the mold, without collapsing it. This gelatin mold was then filled with $0.9 \%$ saline, and the recipient was placed in a $-80^{\circ} \mathrm{C}$ freezer. After the spheres were frozen, they were gently extracted from this mold and embedded in a second $\mathrm{CuSO}_{4}$ gelatin phantom. After the spheres returned to room temperature, a phantom with 4 saline-filled spheric cavities was obtained.

MR images were obtained at this point to measure sphere sizes. After confirming the size of the cavities, we punctured them and performed a coiling procedure by using identical sizes and types of GDCs or the Target coils with packing densities of $15 \%$ and $36 \%$ in the larger and smaller cavities, respectively. The same MR imaging protocol described previously was performed, excluding the phase-contrast and contrast-enhanced sequences. The artifact volume was measured as described previously in the canine experiments. The cavities were re-accessed with a 22-ga-long needle for saline evacuation. The coiled cavities were filled with fresh porcine blood. MR imaging and artifact measurements were repeated. 3D RA was performed to record the coil mass size after each MR imaging session.

\section{Statistical Analysis}

Results are expressed as mean \pm the standard error of the mean. OEFs from longitudinal in vivo studies were analyzed with a repeated-mea- sures ANOVA with a Tukey multiple comparison posttest. OEFs in the phantom models with different environments were compared using a paired Student $t$ test. A value of $P<.05$ was interpreted as statistically significant.

\section{Results}

Twelve canine aneurysms were embolized, and the average packing attenuation was $22.6 \pm 7.7 \%$. Four aneurysms were excluded due to complete or partial aneurysmal thrombosis prior to coil embolization. Representative MR images are provided in Fig 1. Figure 2 presents the $3 \mathrm{D}$ reconstruction of the susceptibility artifacts (Fig 2A) and 3DRA of the coil mass (Fig $2 B)$. OEFs were the largest immediately after coil embolization and showed a dramatic decrease after 1 week postembolization $(P<.01)$. The OEFs continued to gradually decay until approximately 4 weeks after embolization $(P<.001)$, when there was stabilization of the size of the artifacts (Fig 3). At 4 weeks, we found that there was mild coil compaction (average coil mass volume decrease of $7.8 \pm 0.9 \%$ ). However, the artifacts reduced substantially during the same time period, with the OEFs decreasing by $25 \pm 2.8 \%$. At each time point, there was no difference in the OEFs between the different coil families.

Explanted coils from the in vivo aneurysms were examined under SEM to investigate possible galvanic corrosion of the detachment zones, which are constructed from stainless steel (Fig 4). The detachment of the coil is completed by electrolysis of the stainless steel segment. One of the explanted coils had a very small segment of metal exposed $(12 \mu \mathrm{m})$, and minimal pitting was visualized. However, on a spectral analysis, there was no oxide layer. These results support the hypothesis that galvanic corrosion is not responsible for the decrease in susceptibility artifacts. 


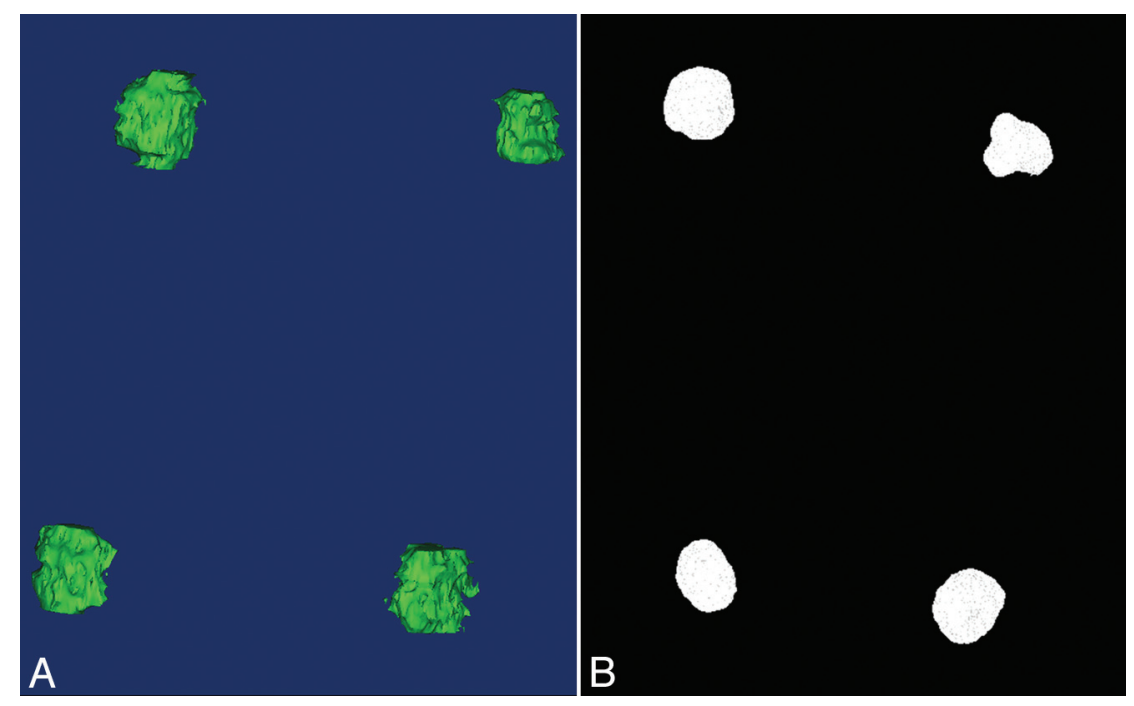

Fig 2. Example of the $3 D$ datasets. $A, 3 D$ reconstruction of the susceptibility artifacts at 14 weeks. $B, 3 D$ RA of the coil masses at 14 weeks. Note slight changes in the subject positioning between the angiography and MR imaging suites.
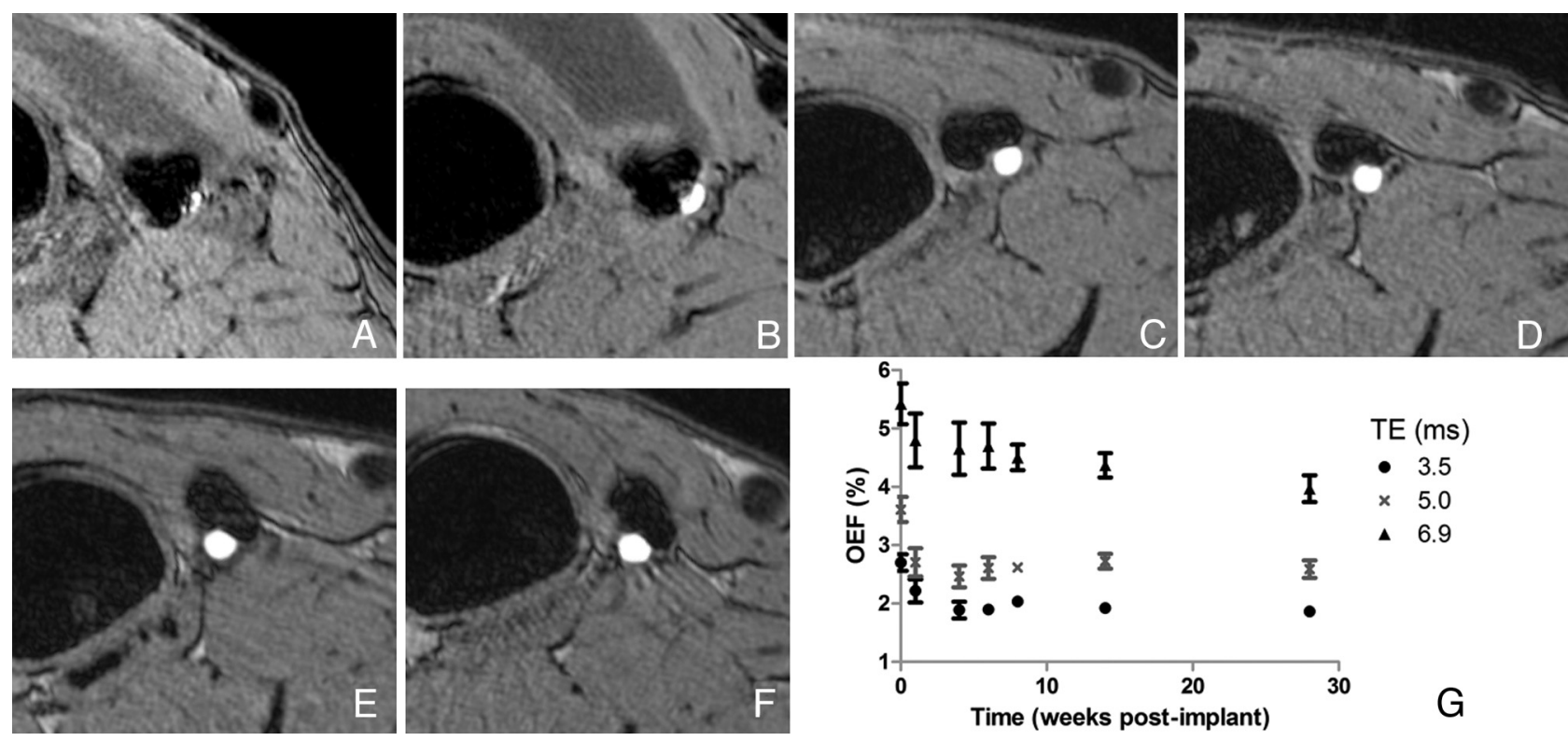

TE (ms)

- 3.5

$\times 5.0$

$\triangle 6.9$

Fig 3. TOF MRA (sequence 2, axial view) of the same aneurysm immediately following coil embolization $(A)$ and at $1(B), 4(C), 8(D), 14(E)$, and $28(F)$ weeks after coil embolization. $G, \mathrm{OEF}$ decreases between zero and approximately 4 weeks, when it becomes stable. Note that the OEF increases with increasing TE. Error bars are the standard error of the mean.

Using the phantom, we explored the relationship between MR imaging artifacts and aneurysm volume difference. There was no difference in OEF between the 3- and 4-mm coiled spheres. The same phantom was used to study the contribution of blood products to the MR imaging artifacts. We had previously measured the artifacts of the coiled cavities with saline. After evacuating the saline and injecting blood, we remeasured the artifacts (Fig 5). From the saline-filled cavity to the blood-filled cavity, there was a $22 \pm 2.3 \%$ increase in susceptibility artifacts $(P<.01)$.

\section{Discussion}

In Latin "susceptibilis" is receptiveness. Magnetic susceptibility is a measure of the extent to which a material may be magnetized in relation to a given applied magnetic field. This is a property of the material that can be classified as diamagnetic, paramagnetic, or ferromagnetic on the basis of their susceptibilities. Susceptibility artifacts are generated by an object in the MR imaging with a higher or lower magnetic susceptibility. ${ }^{24}$ The presence of any object in the MR imaging scanner will cause a distortion of the main magnetic field. The extent of the distortion is determined by the size and the material properties of the object and the MR sequence used. Paramagnetic objects, such as coils, will cause susceptibility artifacts that may be observed as a shift or loss in signal intensity. ${ }^{17}$

The size and shape of susceptibility artifacts are determined not only by the presence of various materials with different magnetic susceptibilities but also by the shape of these materials. In addition, the shape and size of these artifacts are influenced by the magnitude and direction of the readout gradient. Predicting the shape and size of susceptibility artifacts is a complex task that requires multiparameter simulation. 

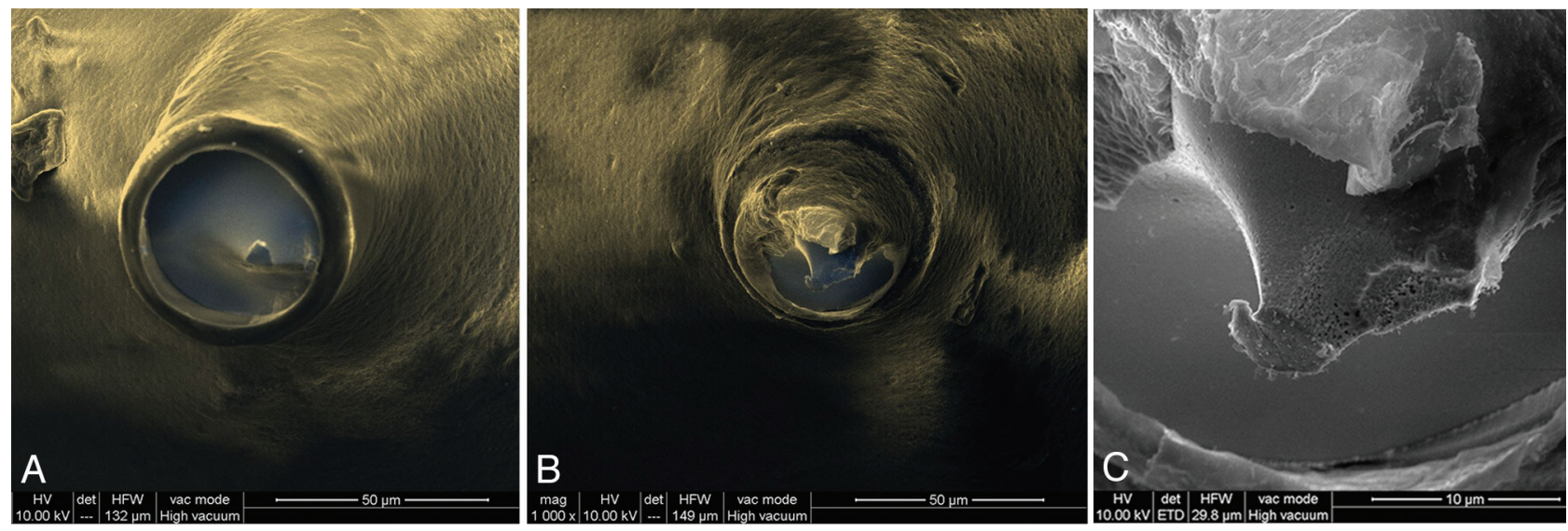

Fig 4. SEM of the detachment zone explanted coils. A, Representative coil with polymer coating sealing the stainless steel, preventing contact with blood products (composite image of backscattered and secondary electrons, original magnification $\times 1126)$. $B$ and $C$, A single coil was discovered with a 12- $\mu$ m segment of stainless steel protruding from the polymer seal that led to minimal exposure of the metal $(B$, composite of backscattered and secondary electrons, original magnification $\times 1000$; $C$, secondary electron image, original magnification $\times 5000)$.

GDC and Target coils are made of platinum alloy. Previous research has been done in vitro to evaluate MR susceptibility artifacts of these coils. Hartman et $\mathrm{al}^{25}$ studied GDC artifacts with the coils contained in a plastic vial filled with saline. In their study, it was concluded that the coils produced minimal susceptibility artifacts in all studied sequences (spin-echo T1, fast spin-echo proton attenuation, T2, inversion recovery, TOF) and they described another type of artifact as a 1-mm rim of high signal intensity immediately adjacent to the coil mass. They also evaluated the MR imaging artifacts of 8 patients who were treated with GDC coils and visually compared the images with those from angiography. They reported that there were minimal artifacts, which did not compromise the evaluation of the perianeurysmal area. These high-signal-intensity-rim artifacts have previously been described in other in vitro and in vivo studies. ${ }^{19,26}$

Derdeyn et $\mathrm{al}^{26}$ compared MR images with DSA images of 26 patients with intracranial aneurysms treated with coiling. There was no subjective signal-intensity loss beyond the expected margins of the aneurysm; however, no quantitative analysis was performed. Gonner et $\mathrm{al}^{15}$ studied an OEF between MRA and DSA images of human coiled aneurysms. However, their OEF was calculated by using the diameter of the artifacts, allowing comparison of 3D MRA with 2D DSA. The overestimation number they described was $2.03 \pm 0.88$ $\mathrm{mm}$ (mean). Although they had different time points at which the MR images were acquired in relation to the coiling procedure, temporal variations of the OEF were not studied. The studies comparing DSA with MRA for follow-up of coiled aneurysm are generally qualitative, comparing parameters such as residual flow within the aneurysm and parent vessel patency. ${ }^{27}$

The susceptibility MR imaging artifacts from coil masses have been long known. The fact that MR imaging overestimates the size of the coil mass is also known. However, the dynamic characteristic of the MR imaging artifacts with time that we observed, to our knowledge, has not yet been described. The decrease in red blood cells and organization of the thrombus inside the aneurysm are coincident with our time points of MR imaging susceptibility artifacts decrease. The histopathology of human coiled aneurysm postmortem spec- imens has been studied by many groups; usually in the first 10 days post-coil embolization, unorganized thrombus, which comprises red blood cells and a fibrin network, can be found. As the thrombus organizes, red blood cells are replaced by fibrinous thrombus, which can be found at around 2-5 weeks. In later time points, fibrovascular tissue is the main finding. ${ }^{28-31}$

Recently, the use of MR imaging, especially TOF sequences, for posttreatment aneurysm surveillance has been increasingly reported. ${ }^{4,32-36}$ Generally, a baseline MR imaging is performed before patient discharge, in the same hospital admission. ${ }^{10}$ These images are then used as a baseline comparison for future follow-ups. If the same phenomena that we observed happen in humans, we may be overestimating the recanalization rates by reading as recanalization what was previously obscured by artifacts. The data presented herein suggest that baseline postembolization MRA should be performed after waiting at least 1 week following the procedure for comparison with future surveillance studies.

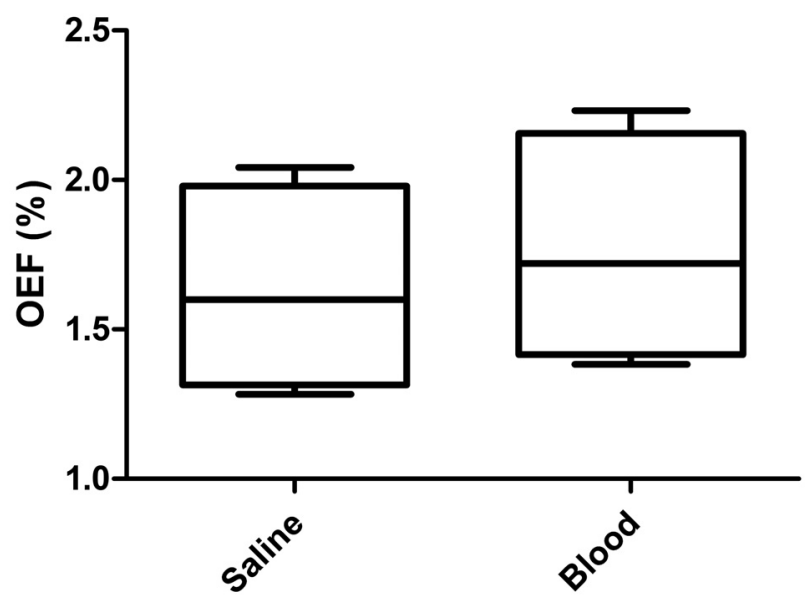

Fig 5. Box-and-whisker plot of the OEF measured in the phantom experiments when the coiled cavity is filled with saline or blood. The presence of blood results in an increase in OEF $(P<.01)$ 


\section{Conclusions}

In this study, we analyzed the MR imaging susceptibility artifacts generated by bare-platinum-coiled aneurysms at different time points. In this animal model, we observed a $25 \%$ decrease in the MR imaging artifacts throughout the first 4 weeks, independent of the coil family used. Various hypotheses were evaluated to explain such phenomena. We found that the temporal evolution of thrombus organization within the aneurysm is consistent with reduction of MR imaging susceptibility artifacts. Further research is necessary to evaluate susceptibility artifacts reduction clinically.

Disclosures: Sarena Carniato—UNRELATED: Employment: Stryker. Ajay Wakhloo-UNRELATED: Consultancy: Stryker, ev3, Codman, Surpass, Comments: fee per hour consultation; Grants/Grants Pending: National Institutes of Health, ${ }^{*}$ Philips, ${ }^{*}$ Stock/Stock Options: Surpass Medical, Comments: holds stock in Surpass Medical. Matthew Gounis-RELATED: Grant: Stryker, ${ }^{*}$ Comments: research funded by a grant from Stryker Neurovascular (principal investigator: Gounis); UNRELATED: Consultancy: Codman, Micrus, Soteira, Comments: fee per hour of consultation. Grants/Grants Pending: National Institutes of Health, ${ }^{*}$ Codman Neurovascular, ${ }^{*}$ Concentric Medical, ${ }^{*}$ eV3 Neurovascular, ${ }^{*}$ Neuravi, ${ }^{*}$ Neurointerventional Therapeutics, ${ }^{*}$ Sanofi-aventis, ${ }^{*}$ Soteira, ${ }^{*}$ Thrombolytic Science, ${ }^{*}$ *Money paid to the institution.

\section{References}

1. Molyneux AJ, Kerr RS, Yu LM, et al. International Subarachnoid Aneurysm Trial (ISAT) of neurosurgical clipping versus endovascular coiling in 2143 patients with ruptured intracranial aneurysms: a randomised comparison of effects on survival, dependency, seizures, rebleeding, subgroups, and aneurysm occlusion. Lancet 2005;366:809-17

2. Raymond J, Guilbert F, Weill A, et al. Long-term angiographic recurrences after selective endovascular treatment of aneurysms with detachable coils. Stroke 2003;34:1398-403

3. Byrne JV, Sohn MJ, Molyneux AJ, et al. Five-year experience in using coil embolization for ruptured intracranial aneurysms: outcomes and incidence of late rebleeding. J Neurosurg 1999;90:656-63

4. van Rooij WJ, Sluzewski M. Opinion: imaging follow-up after coiling of intracranial aneurysms. AJNR Am J Neuroradiol 2009;30:1646-48

5. Gallas S, Januel AC, Pasco A, et al. Long-term follow-up of 1036 cerebral aneurysms treated by bare coils: a multicentric cohort treated between 1998 and 2003. AJNR Am J Neuroradiol 2009;30:1986-92

6. Connors JJ 3rd, Sacks D, Furlan AJ, et al. Training, competency, and credentialing standards for diagnostic cervicocerebral angiography, carotid stenting, and cerebrovascular intervention: a joint statement from the American Academy of Neurology, the American Association of Neurological Surgeons, the American Society of Interventional and Therapeutic Neuroradiology, the American Society of Neuroradiology, the Congress of Neurological Surgeons, the AANS/CNS Cerebrovascular Section, and the Society of Interventional Radiology. Neurology 2005;64:190-98

7. Willinsky RA, Taylor SM, TerBrugge K, et al. Neurologic complications of cerebral angiography: prospective analysis of 2,899 procedures and review of the literature. Radiology 2003;227:522-28

8. Kaufmann TJ, Huston J III, Mandrekar JN, et al. Complications of diagnostic cerebral angiography: evaluation in 19,826 consecutive patients. Radiology 2007;243:812-19

9. Mamourian AC, Young H, Stiefel MF. Cumulative radiation dose in patients admitted with subarachnoid hemorrhage: a prospective study using a selfdeveloping film badge. AJNR Am J Neuroradiol 2010;31:1787-90. Epub 2010 Jul 1

10. Wallace RC, Karis JP, Partovi S, et al. Noninvasive imaging of treated cerebral aneurysms. Part I. MR angiographic follow-up of coiled aneurysms. AJNR Am J Neuroradiol 2007;28:1001-08

11. Bakker NA, Westerlaan HE, Metzemaekers JD, et al. Feasibility of magnetic resonance angiography (MRA) follow-up as the primary imaging modality after coiling of intracranial aneurysms. Acta Radiol 2010;51:226-32

12. Ferre JC, Carsin-Nicol B, Morandi X, et al. Time-of-flight MR angiography at $3 \mathrm{~T}$ versus digital subtraction angiography in the imaging follow-up of 51 intracranial aneurysms treated with coils. Eur J Radiol 2009;72:365-69
13. Urbach H, Dorenbeck U, von Falkenhausen M, et al. Three-dimensional timeof-flight MR angiography at $3 \mathrm{~T}$ compared to digital subtraction angiography in the follow-up of ruptured and coiled intracranial aneurysms: a prospective study. Neuroradiology 2008;50:383-89

14. Schmalbrock P, Yuan C, Chakeres DW, et al. Volume MR angiography: methods to achieve very short echo times. Radiology 1990;175:861-65

15. Gonner F, Heid O, Remonda L, et al. MR angiography with ultrashort echo time in cerebral aneurysms treated with Guglielmi detachable coils. AJNR Am J Neuroradiol 1998;19:1324-28

16. Reinhardt J, Nguyen-Trong TH, Hahnel S, et al. Magnetic resonance imaging of stents: quantitative in vitro examination at 3 Tesla. $Z$ Med Phys 2009;19: $278-87$

17. Walker MT, Tsai J, Parish T, et al. MR angiographic evaluation of platinum coil packs at 1.5T and 3T: an in vitro assessment of artifact production-technical note. AJNR Am J Neuroradiol 2005;26:848-53

18. Kakeda S, Korogi Y, Hiai Y, et al. MRA of intracranial aneurysms embolized with platinum coils: a vascular phantom study at $1.5 \mathrm{~T}$ and 3T. J Magn Reson Imaging 2008;28:13-20

19. Hennemeyer CT, Wicklow K, Feinberg DA, et al. In vitro evaluation of platinum Guglielmi detachable coils at $3 \mathrm{~T}$ with a porcine model: safety issues and artifacts. Radiology 2001;219:732-37

20. German W, Black S. Experimental production of carotid aneurysms. N Engl JMed 1954;250:104-06

21. Strother CM, Graves VB, Rappe A. Aneurysm hemodynamics: an experimental study. AJNR Am J Neuroradiol 1992;13:1089-95

22. Wakhloo AK, Schellhammer F, de Vries J, et al. Self-expanding and balloonexpandable stents in the treatment of carotid aneurysms: an experimental study in a canine model. AJNR Am J Neuroradiol 1994;15:493-502

23. Piotin M, Daughman B, Mounayer C, et al. Ellipsoid approximation versus $3 D$ rotational angiography in the volumetric assessment of intracranial aneurysms. AJNR Am J Neuroradiol 2006;27:839-42

24. Oehler MC, Schmalbrock P, Chakeres D, et al. Magnetic susceptibility artifacts on high-resolution MR of the temporal bone. AJNR Am J Neuroradiol 1995; 16: 1135-43

25. Hartman J, Nguyen T, Larsen D, et al. MR artifacts, heat production, and ferromagnetism of Guglielmi detachable coils. AJNR Am J Neuroradiol 1997; 18:497-501

26. Derdeyn CP, Graves VB, Turski PA, et al. MR angiography of saccular aneurysms after treatment with Guglielmi detachable coils: preliminary experience. AJNR Am J Neuroradiol 1997;18:279-86

27. Yamada N, Hayashi K, Murao K, et al. Time-of-flight MR angiography targeted to coiled intracranial aneurysms is more sensitive to residual flow than is digital subtraction angiography. AJNR Am J Neuroradiol 2004;25:1154-57

28. Bavinzski G, Talazoglu V, Killer M, et al. Gross and microscopic histopathological findings in aneurysms of the human brain treated with Guglielmi detachable coils. J Neurosurg 1999;91:284-93

29. Killer M, Arthur AS, Barr JD, et al. Histomorphology of thrombus organization, neointima formation, and foreign body response in retrieved human aneurysms treated with Hydrocoil devices. J Biomed Mater Res B Appl Biomater 2010;94:486-92

30. Ishihara S, Mawad ME, Ogata $\mathrm{K}$, et al. Histopathologic findings in human cerebral aneurysms embolized with platinum coils: report of two cases and review of the literature. AJNR Am J Neuroradiol 2002;23:970-74

31. Groden C, Hagel C, Delling G, et al. Histological findings in ruptured aneurysms treated with GDCs: six examples at varying times after treatment. $A J N R$ Am J Neuroradiol 2003;24:579-84

32. Shankar JJ, Lum C, Parikh N, et al. Long-term prospective follow-up of intracranial aneurysms treated with endovascular coiling using contrastenhanced MR angiography. AJNR Am J Neuroradiol 2010;31:1211-15

33. Sprengers ME, van Rooij WJ, Sluzewski M, et al. MR angiography follow-up 5 years after coiling: frequency of new aneurysms and enlargement of untreated aneurysms. AJNR Am J Neuroradiol 2009;30:303-07

34. Anzalone N, Righi C, Simionato F, et al. Three-dimensional time-of-flight MR angiography in the evaluation of intracranial aneurysms treated with Guglielmi detachable coils. AJNR Am J Neuroradiol 2000;21:746-52

35. Kaufmann TJ, Huston J 3rd, Cloft HJ, et al. A prospective trial of 3T and 1.5T time-of-flight and contrast-enhanced MR angiography in the follow-up of coiled intracranial aneurysms. AJNR Am J Neuroradiol 2010;31:912-18

36. Pierot L, Delcourt C, Bouquigny F, et al. Follow-up of intracranial aneurysms selectively treated with coils: prospective evaluation of contrast-enhanced MR angiography. AJNR Am J Neuroradiol 2006;27:744-49 